\title{
EL ECODESARROLLO COMO CONCEPTO PRECURSOR DEL DESARROLLO SUSTENTABLE Y SU INFLUENCIA EN AMERICA LATINA ${ }^{1}$
}

\author{
The Ecodevelopment as concept precursor \\ of sustainable development and its influence in Latin America
}

Fernando Estenssoro*

\begin{abstract}
RESUMEN
El artículo trabaja sobre la hipótesis de que en el inicio del debate político ambiental mundial, en los años 70, inmediatamente surgió una fuerte polémica Norte-Sur respecto de cómo entender esta problemática. Los países del Sur, insistieron que la protección del Medio Ambiente y la consecución del pleno desarrollo para el mundo subdesarrollado eran dos aspectos indisolubles al momento de abordar el tema de la crisis ambiental. En este sentido, un primer concepto que unió esta dos ideas de medio ambiente y desarrollo fue el de ecodesarrollo, profundizado y teorizado por Ignacy Sachs, pero inmediatamente recogido y socializado por los intelectuales e instituciones de América Latina que participaban del debate. En este sentido, el ecodesarrollo fue precursor del posterior concepto de desarrollo sustentable elaborado en 1987 por la Comisión Brundtland, y que unió definitivamente las ideas de medio ambiente y desarrollo.
\end{abstract}

Palabras clave: Medio Ambiente, América Latina, ecodesarrollo, Norte-Sur, política ambiental.

\footnotetext{
${ }^{1}$ Este artículo es producto del proyecto $\mathrm{N}^{\circ} 1110424$ del Fondo Nacional de Desarrollo Científico y Tecnológico (Fondecyt), titulado:"Recepción e impacto de la ecología política en los Partidos Comunista y Socialista de Chile. Un estudio de historia de las ideas políticas en el cambio de siglo. 1980- 2010".

* Instituto de Estudios Avanzados, Universidad de Santiago de Chile. Santiago, Chile. Correo electrónico: fernando.estenssoro@usach.cl
}

Artículo recibido el 20 de agosto de 2013. Aceptado el 7 de julio de 2014. 


\begin{abstract}
The article works on the assumption that at the beginning of global environmental policy debate in the 1970s, immediately arose a strong North-South debate on how to understand this problem. The South, insisted that protecting the environment and achieving full development for the underdeveloped world were two inseparable aspects when addressing the issue of environmental crisis. In this sense, a concept first joined these two ideas of environment and development was the eco-development, deepened and theorized by Ignacy Sachs, but it was immediately picked up and socialized by Latin American intellectuals and institutions participating in the debate. In this sense, the ecodevelopment was precursor to the later concept of sustainable development, generated in 1987 by the Brundtland Commission, which permanently joined to the ideas of environment and development.
\end{abstract}

Keywords: Environment, Latin America, ecodevelopment, North-South, environmental policy.

\title{
INTRODUCCIÓN
}

El tema del medio ambiente se instaló formalmente en la agenda política mundial tras la realización de la Conferencia sobre el Medio Humano, citada por la Organización de las Naciones Unidas y realizada en Estocolmo en 1972. En esa oportunidad, tanto en sus documentos de convocatoria como en su declaración final, junto con la creación del Programa de Naciones Unidas para el Medio Ambiente (PNUMA), quedó estipulado que el mundo enfrentaba una crisis ambiental global producto del modo de vida predominante que había alcanzado el ser humano y caracterizado, en términos genéricos, como la "Civilización Industrial”2. Se señaló en esa oportunidad que, por ignorancia o por inacción, se estaban causando dańos y/o se podían causar daños irreparables al planeta del cual dependía la vida y el bienestar del ser humano. Estos problemas, por ser de alcance regional y/o mundial, iban a requerir de una amplia colaboración entre las naciones del mundo para su solución, según se podía leer en la Declaración Final de la Conferencia:

\footnotetext{
${ }^{2}$ En el documento base de esta conferencia titulado, Una Sola Tierra, se podía leer: "Se enfrenta una crisis ambiental global que pone en riesgo la vida del ser humano y del planeta. [...] Nuestras bruscas y vastas aceleraciones -en el crecimiento demográfico, en el uso de la energía y de nuevos materiales, en la urbanización, en los ideales de consumo y en la contaminación resultante- han colocado al hombre tecnológico en la ruta que podía alterar, en forma peligrosa, y quizá irreversible, los sistemas naturales de su planeta, de los cuales depende su supervivencia biológica [...] En pocas palabras, los dos mundos del hombre -la biósfera de su herencia y la tecnósfera de su creación- se encuentran en desequilibrio y, en verdad, potencialmente, en profundo conflicto. Y el hombre se encuentra en medio" (Ward y Dubos, 1984: 39-49).
} 
Hay un número cada vez mayor de problemas relativos al medio que, por ser de alcance regional o mundial o por repercutir en el ámbito internacional común, requerirán de una amplia colaboración entre las naciones y la adopción de medidas por las organizaciones internacionales en interés de todos. La Conferencia encarece a los gobiernos y a los pueblos a que aúnen sus esfuerzos para preservar y mejorar el medio humano en beneficio del hombre y de su posteridad (Grinberg, 1999: 181).

Por lo tanto, desde Estocolmo 1972, esta idea de crisis ambiental, con todas sus complejidades y contradicciones fue inscrita formalmente en la agenda política mundial y, desde aquí en adelante, los países y pueblos del mundo deberían abocarse a diseñar y aplicar las políticas concretas destinadas a su superación.

Sin embargo, la concreción exitosa de esta Conferencia no fue algo fácil y de hecho estuvo cerca de fracasar, entre otras razones, por la confrontación de diferentes perspectivas para tratar este tema entre los países desarrollados y los países en vías de desarrollo.

La convocatoria a esta Conferencia era una iniciativa de los países altamente desarrollados e industrializados o Primer Mundo, quienes enfatizaban sus particulares puntos de vista frente a la problemática ambiental, como bien recordó el propio Maurice Strong, Secretario General de la Conferencia de Estocolmo 1972, al señalar que fue en "los países industrializados donde la preocupación por la contaminación creó la idea original de la Conferencia de Estocolmo" (Strong, 1983: 247). En este sentido, la perspectiva primermundista traía una fuerte crítica al propio proceso de desarrollo y crecimiento económico de sus sociedades hiperindustrializadas que, precisamente, los había convertido en Primer Mundo, pero que, a la vez, había causado enormes daños al ambiente y a sus ecosistemas. Por este motivo, enfatizaban las variables de contaminación y polución industrial de todo tipo, y por sobre todo, el peligro que veían en el crecimiento demográfico del Tercer Mundo que, según su perspectiva de países centrales, amenazaba con arrasar los recursos del planeta. Además, también expresaban el temor de que la industrialización que pretendía el Tercer Mundo terminara por deteriorar definitivamente los ecosistemas naturales que aún quedaban en el globo, poniendo en riesgo la propia supervivencia de la biosfera ${ }^{3}$.

${ }^{3}$ A modo de ejemplo, recordemos que ya en 1948 se habían publicado los libros, de gran impacto, de los científicos estadounidenses William Vogt, Road to Survival y Fairfield Osborn, Our Plundered Planet, donde, lograron popularizar la idea de crisis final de la civilización por el agotamiento de los recursos naturales ante el aumento de las bocas que alimentar. Igualmente, en 1967 los hermanos y biólogos estadounidenses William y Paul Paddock publicaron Famine, 1975!: America's decision: Who will survive?, señalando que los países industrializados no deberían ayudar a los países subdesarrollados a superar sus hambrunas, ya que a raíz del alto crecimiento demográfico de los países pobres los recursos que se les entregaban por parte del mundo desarrollado no eliminarían las causas de su miseria sino que ayudarían a que estos siguieran reproduciéndose. Al ańo siguiente, en 1968, Paul Erhlich popularizó la idea de "explosión demográfica” en su libro The Population Bomb. También en 1968, se creó el Club de Roma, que en 1972, y en consonancia con la Conferencia de Estocolmo, lanzó su conocido informe Los Limites del Crecimiento (Estenssoro, 2009; Mahrane, 2012). 
Esta perspectiva primermundista para enfrentar el tema de la crisis ambiental, provocó una profunda desconfianza en los países en vías de desarrollo o Tercer Mundo respecto de las verdaderas intenciones que se perseguía con esta Conferencia, como bien señala Roberto Guimaraes:

El énfasis en Estocolmo estaba puesto en los aspectos técnicos de la contaminación provocada por la industrialización acelerada, por la explosión demográfica y por la intensificación del proceso de crecimiento urbano, todo lo cual imprimía un carácter nítidamente primer-mundista a la reunión. No debería sorprender el alto grado de resistencia demostrado por los países del Tercer Mundo en aquel entonces. Como lo resumió el representante del gobierno de la India en una reunión preparatoria a Estocolmo [...] «Los ricos se preocupan del humo que sale de sus autos; a nosotros nos preocupa el hambre» (Guimaraes, 1992: 87-88).

En otras palabras, el Sur del mundo sospechaba que los países desarrollados estaban orientando los esfuerzos del sistema internacional a priorizar la resolución de aquellos temas que venían afectando la calidad de vida de sus ya opulentas sociedades, dejando de lado los esfuerzos por superar el subdesarrollo de la mayoría de la humanidad. $\mathrm{Al}$ respecto, se debe recordar que los principales esfuerzos del Tercer Mundo en el sistema internacional, desde el término de la Segunda Guerra Mundial en adelante, estaban orientados a implementar procesos de desarrollo, crecimiento y modernización social y productiva, y para esto buscaban cambiar las relaciones desiguales del comercio e intercambio internacional, implementar estrategias de industrialización y/o tener acceso a las nuevas tecnologías, entre otras múltiple medidas ${ }^{4}$.

Esta situación de desconfianza desató una intensa polémica Norte-Sur, sobre las problemáticas del desarrollo que tanto importaba a los países subdesarrollados, como bien señala Paolo Bifani, al recordar que en los primeros años de iniciado el debate ambiental:

Se explicitaba una violenta crítica del concepto de desarrollo dominante, en el cual prevalecía la idea de crecimiento: para muchos desarrollo era (y aún es) sinónimo

\footnotetext{
${ }^{4}$ Recordemos, para el caso de AML, todo el aporte a las teorías del desarrollo que significó la creación en 1948 de la Comisión Económica para América Latina (CEPAL), desde donde surgieron planteamientos tales como la teoría de la Dependencia, y la estrategia de industrialización sustitutiva que se implementó desde los ańos cincuenta en adelante. Igualmente se debe recordar que en 1962 se creó el Instituto Latinoamericano de Planificación Económica y Social (ILPES), destinado a educar a los funcionarios públicos de la región en materias propias de las teorías del desarrollo. También se debe recordar que en 1964 se creó la Conferencia de Naciones Unidas sobre Comercio y Desarrollo (UNCTAD), cuyos objetivos se orientaban a maximizar las oportunidades comerciales, de inversión y desarrollo de los países en vías de desarrollo así como la asistencia para que sus economías se desarrollaran (Gutiérrez y González, 2010).
} 
de crecimiento económico. El debate se polarizó entre una posición extrema que identificaba crecimiento económico con desarrollo y veía, en el mismo, una de las causas fundamentales de la crisis de aquella época: energética, de alimentos, ambiental. En esa perspectiva, el crecimiento y desarrollo era negativo tenía un carácter cancerígeno y la sobrevivencia de la especie humana y del planeta requería que el crecimiento, tanto poblacional como económico, terminara, el objetivo era el crecimiento cero. Frente a esta posición de claro carácter neomalthusiana, otras propusieron revisar el concepto de desarrollo y explicitar sus múltiples dimensiones, entre ellas la ambiental. Este era la situación del debate sobre el desarrollo al iniciarse la Conferencia de las Naciones Unidas sobre el Medio Humano en Estocolmo en 1972. Los años sesenta y setenta fueron testigos de una crítica despiadada del desarrollo (crecimiento) visto por algunos como causa primera del deterioro ambiental(Bifani, 1999: 105).

\section{LA INICIATIVA DEL BOICOT DEL TERCER MUNDO Y EL INFOME DE FOUNEX}

Esta desconfianza de los representantes de los países en vías de desarrollo respecto de la forma cómo el mundo desarrollado enfocaba el problema ambiental hizo crisis a inicios de 1971, tras la segunda reunión preparatoria de la Conferencia de Estocolmo, cuando los representantes del Sur comenzaron a conversar la posibilidad de boicotear el evento.

Strong, que había sido puesto al tanto de esta situación por el embajador de Yugoslavia, debió realizar un intenso lobby con los representantes del Tercer Mundo para evitar que todos sus esfuerzos por realizar una conferencia exitosa fracasaran estrepitosamente. Entre las distintas acciones que realizó, fue clave la convocatoria a una reunión ad hoc, en el pueblito suizo de Founex, a un selecto grupo de 27 personalidades de renombre mundial, principalmente representantes del Sur y expertos en temas del desarrollo, a fin de alcanzar un acuerdo y consensuar un punto de vista que recogiera los intereses del Tercer Mundo, referidos a superar el subdesarrollo, miseria y pobreza y unirlos al tema de la crisis ambiental (Tamames, 1979; Engfeldt, 2009; Sánchez, 2011). Así, entre el 4 y 12 de junio de 1971, se reunieron expertos en temas del desarrollo, economía y relaciones internacionales, vinculados a la Conferencia de las Naciones Unidas Sobre el Comercio y Desarrollo (UNCTAD), al Acuerdo General sobre Aranceles Aduaneros y Comercio (GATT) y a la FAO, entre otras agencias 5 , y emitieron el Informe de Founex en el cual, por primera vez, se unían dos ideas que hasta ese momento aparecían como

\footnotetext{
${ }^{5}$ Tales como Ignacy Sachs, Samir Amin, Enrique Iglesias, Felipe Herrera, William Kapp, Miguel Ozorio de Almeida, Pitambar Pant, Jan Tinbergen, Shigeto Tsuru, entre otros. (http://www. mauricestrong.net). [Consultado el: 23 de octubre de 2012].
} 
contradictorias: la idea de proteger el medio ambiente y la idea de alcanzar el pleno desarrollo. Además, también se señaló que la crisis ambiental, en gran parte se debía el estilo de desarrollo de los países altamente industrializados y que, a diferencia del Primer Mundo, en el Tercer Mundo, la pobreza y la miseria eran la mayor expresión de la crisis ambiental:

Puede afirmarse que, en gran medida, el actual interés en las cuestiones relacionadas con el medio ambiente ha tenido su origen en los problemas experimentados por los países industrialmente adelantados. Estos problemas son de por sí, en gran parte, el resultado de un nivel elevado de desarrollo económico [...] Estas perturbaciones han llegado a alcanzar tales proporciones que en muchos sitios constituyen ya un grave peligro para la salud y el bienestar humanos [...] Sin embargo, los principales problemas ambientales de los países en desarrollo son básicamente diferentes de los que se perciben en los países industrializados. Son principalmente problemas que tienen su raíz en la pobreza y la propia falta de desarrollo de sus sociedades. En otras palabras, son problemas de pobreza rural y urbana [...] Por estas razones, la preocupación por el medio ambiente no debe debilitar, y no es preciso que lo haga, el compromiso de la comunidad mundial -tanto de los países en desarrollo como de los industrializados- de dedicarse a la tarea principalísima de desarrollar las regiones más atrasadas del mundo. Por el contrario, subraya la necesidad no sólo de comprometerse plenamente a alcanzar las metas y objetivos del segundo decenio para el desarrollo, sino también redefinirlas a fin de atacar la miseria que es el aspecto más importante de los problemas que afligen al medio ambiente de la mayoría de la humanidad (El Informe Founex, en Marino de Botero y Tokatlian, 1983: 51-85).

Este Informe de Founex, donde se unían las ideas de medio ambiente y desarrollo, fue incorporado como documento base a la Conferencia de Estocolmo y permitió que esta siguiera su curso pre-establecido. De esta forma se pudieron realizar las dos últimas reuniones preparatorias, en septiembre de 1971 y marzo de 1972 en Nueva York, para llegar finalmente en junio de 1972 a la gran Conferencia de la capital sueca.

\section{IGNACY SACH Y EL ECODESARROLLO}

Sin embargo, terminada la Conferencia esta discusión continuó. En los países del Tercer Mundo en general y América Latina (AML) en particular, así como en grupos de intelectuales progresistas del Primer Mundo, siguieron desarrollando y profundizando la idea de unir medio ambiente y desarrollo. En este sentido, uno de los conceptos más interesantes que surgieron, fue el de ecodesarrollo. 
El concepto de ecodesarrollo fue utilizado por primera vez por Maurice Strong que, inspirado en el Informe de Founex, lo planteó en la primera reunión del Consejo Consultivo del PNUMA llevada a cabo en Ginebra en junio de 1973, para graficar "una forma de desarrollo económico y social en cuya planificación debe considerarse la variable medio ambiente" (Sánchez et al., 1978: 12). Para Strong, el desarrollo y el medio ambiente no solo no se oponían, sino que constituían dos aspectos diferentes del mismo concepto. Lo que realmente estaba en juego era "la gestión racional de los recursos con el objeto de mejorar el hábitat global del hombre y asegurar una calidad de vida mejor de todos los seres humanos. Una vez más, es el concepto de desarrollo el que, de esta manera se amplía y se hace más realista..."(Strong, citado en Herzer; 1977: 214).

Sin embargo, quien desarrolló en profundidad este concepto fue el economista polaco, naturalizado francés y posteriormente brasileño, Ignacy Sachs, que en la segunda mitad de los años sesenta se desempeñaba como catedrático del École des Hautes Études en Sciences Sociales. De acuerdo con Iván Restrepo, Sachs expuso el sentido de este concepto por primera vez en el seminario "Medio Ambiente y Desarrollo: Estrategias para el Tercer Mundo", que fue organizado por la Escuela Superior de Economía del Instituto Politécnico en el Colegio Nacional de Economistas de México, en agosto de 1973 (Restrepo, 1976: 9).

$\mathrm{Al}$ año siguiente, en 1974, aparecieron publicados sus planteamientos en el artículo "Ambientes y estilos de desarrollo", donde se señalaba que era posible un desarrollo social continuo en armonía con el medio ambiente, pero para esto era necesario entender que el ambiente era una dimensión del desarrollo y por esto debía ser asimilado en todos los niveles de decisión, lo que obligaba a replantearse las definiciones de desarrollo hasta ese momento dominantes y entender que podían existir múltiples formas del mismo y, por lo tanto, no cabía proponer una sola forma. Particularmente pensando en el desarrollo del Tercer Mundo y los ecosistemas tropicales (si bien no únicamente ellos), Sachs propuso que el ecodesarrollo era una forma de desarrollo adaptado a las realidades eco-sistémicas de cada región o eco-región:

Dada la complejidad del tema y las múltiples formas posibles de combinación de las variables operativas pertinentes, no cabe proponer una sola estrategia de desarrollo. Esto nos lleva a la búsqueda de opciones futuras. El concepto del ecodesarrollo surge de estas consideraciones generales. En efecto, con él se aspira a definir un estilo de desarrollo particularmente adaptado a las regiones rurales del Tercer Mundo [...] es un estilo de desarrollo que busca con insistencia en cada ecorregión soluciones específicas a los problemas particulares, habida cuenta de los datos ecológicos, pero también culturales, así como de las necesidades inmediatas, pero también de las de largo plazo. Así, el ecodesarrollo actúa con 
criterios de progreso relativos, referentes a cada caso, y en el desempeña un papel importante la adaptación al medio, postulada por los antropólogos. Sin negar la significación de los intercambios [...] el ecodesarrollo trata de reaccionar contra la moda predominante de las soluciones pretendidamente universales y las fórmulas maestras (Sachs, 1974: 363, 364).

\section{Ecodesarrollo como respuestas a las tesis del Club de Roma y el crecimiento cero}

Los planteamientos de Sachs se inscribían dentro de la intensa polémica que desató el primer informe del Club de Roma, Los Limites del Crecimiento, y sus postulados de crecimiento cero. Recordemos que este estudio dirigido, por Dennis Meadows y su equipo del Instituto Tecnológico de Massachusetts (MIT), había concluido con su célebre pronóstico que predecía el fin de la civilización si no se revertía la gravedad del problema de la contaminación industrial y si no se estabilizaba el crecimiento económico y de la población mundial, en un punto igual a cero (crecimiento cero), dado que el planeta tenía límites físicos infranqueables que no permitían continuar y sostener el crecimiento y explotación de los recursos naturales tal cual se venía dando, así como tampoco se podía seguir con la industrialización del planeta, y para lo cual llamaron a generar un nuevo orden mundial que evitara el desastre. Si no se modifican las tendencias actuales en cuanto a crecimiento de la población mundial, industrialización, contaminación, producción alimentaria y agotamiento de los recursos, alcanzaremos el límite de crecimiento de este planeta en el transcurso de los próximos cien años. El resultado más probable será una repentina e incontrolable caída de la población y la capacidad industrial.

Es posible alterar estas tendencias y establecer una condición de estabilidad ecológica y económica que sea sostenible largamente en el futuro. El estado de equilibrio global puede ser diseñado de tal forma que las necesidades básicas de cada persona en la tierra sean satisfechas y cada persona tenga una oportunidad igual de realizar su potencial humano individual (Meadows et al., 1972: 23, 24) ${ }^{6}$.

Sachs, al igual que muchos especialistas latinoamericanos (entre otros) criticaron este informe. Para él la urgencia neomalthusiana y catastrofista de los Limites no sólo eran un error sino que servían para nutrir ideologías altamente discutibles, que iban desde el polo antindustrial y antitecnológico radical y su propuesta de que la sociedad volviera a un utópico estado de naturaleza, hasta el otro extremo representado por las tesis de depositar la solución de la crisis ambiental en manos de las empresas multinacionales:

\footnotetext{
${ }^{6}$ La traducción es del autor.
} 
El planteamiento de Meadows involucra una banalidad: si el medio es finito y en este medio una población tiene crecimiento exponencial -más días menos díasvendrá el momento en que aparecerá el límite. Para esto no son necesarias ni computadoras ni modelos. Es una verdad elemental. Todo el problema es cuándo vendrá ese límite, y creo que en términos de impacto ideológico y político, la urgencia que el libro proclama es, quizá, su aspecto más debatible y más importante [...] ¿Cuáles son, por tanto, las implicaciones ideológicas de esta urgencia? Creo que el tema ha sido abordado por corrientes políticas muy distintas y hay, por lo menos, dos grupos bastante opuestos que están apoyándose en los argumentos de Meadows y de Paul Ehrlich, para proponer dos cosas totalmente distintas. De un lado, hay grupos utopistas de izquierda que están sugiriendo un retorno a la naturaleza, a la Rousseau [...] Por otro lado, hay tentativas de interpretar esta problemática como una fase de crisis, por lo que es preciso apoyarse en las organizaciones más capaces; por supuesto, estos organismos son las empresas multinacionales (Sachs, 1976: 13, 14).

Por el contrario, para Sachs, no se trataba de detener el desarrollo sino que de reorientarlo en una perspectiva ecológica y sana ambientalmente. En este sentido, el ecodesarrollo buscaba un proceso de desarrollo humanista consciente de su interdependencia con la naturaleza y, para lo cual postulaba colocar el avance científico-técnico al servicio de la protección de los ecosistemas, o ecotécnica. Esto significaba que había que reorientar la técnica y la ciencia en apoyo al ecodesarrollo en vez de ponerlas al servicio de una lógica de acumulación capitalista creciente, que no sólo alienaba al propio ser humano, sino que, también, destruía la naturaleza y envenenaba el ambiente. De esta forma, el perfeccionamiento de la ecotécnica estaba "llamado a ocupar un lugar muy importante en las estrategias del ecodesarrollo, debido a que la compatibilización de objetivos diversos -económicos, sociales, ecológicos- puede realizarse adecuadamente a este nivel; el cambio técnico surge como la variable multidimensional por excelencia en el proceso de la planificación". Por lo tanto, el ecodesarrollo, "sin caer en un ecologismo a ultranza, sugiere, al contrario, que siempre es posible un esfuerzo creador para aprovechar el margen de libertad que ofrece el medio, por grandes que sean los escollos climáticos y naturales" (Sachs, 1974: 364).

Posteriormente, en otros escritos, señalará que el ecodesarrollo era "un desarrollo socialmente deseable, económicamente viable, y ecológicamente prudente" (Sachs, 1981: 11). 


\section{Ecodesarrollo como concepto precursor del desarrollo Sustentable}

En este sentido, el propio Sachs explica que el ecodesarrollo estaba totalmente inspirado en el espíritu del Informe de Founex, donde se unieron las ideas de medio ambiente y desarrollo.

Al respecto también recuerda que en el debate ambiental suscitado con motivo de la Conferencia de Estocolmo, se enfrentaban dos tendencias diametralmente opuestas. Por una parte los defensores del crecimiento a cualquier precio, que percibían el tema ambiental como un capricho de "burgueses ociosos", o como un obstáculo "al avance al proceso de industrialización de los países del hemisferio Sur". En el otro extremo, estaban los "catastrofistas que anunciaban el apocalipsis para el día siguiente" y que a su vez se dividían entre quienes pregonaban el fin inminente de la civilización como "consecuencia del agotamiento de los recursos naturales", y quienes predecían la catástrofe como "consecuencia de aumento de la población", pero ambos grupos coincidían en la urgencia por detener el crecimiento demográfico y económico mundial (Sachs, 1998: 161).

Frente a estas dos posturas opuestas, surgió el Informe de Founex, que tuvo el buen sentido de no dar la razón a ninguna de ellas, tratando, a su vez de recoger los aspectos positivos de ambas. O sea, fue una línea intermedia entre "el ecologismo absoluto y el economicismo arrogante", y que postulaba "un desarrollo orientado por el principio de la justicia social en harmonía con la naturaleza, y no a través de su conquista" (Ibid: 162).

Por cierto, que el crecimiento económico era necesario, pero "debería asumir un perfil diferente de aquel caracterizado como "crecimiento salvaje, y por encima de todo, sus frutos deberían ser utilizados y repartidos de otra manera". Por lo tanto, para Sachs el ecodesarrollo era la "armonización de objetivos sociales, ambientales y económicos" (Ibíd.).

En este sentido, parte importante de los postulados del ecodesarrollo fueron recogidos en la fórmula que sintetizó el conocido concepto de Sustainable Development, que en castellano se ha traducido indistintamente como Desarrollo Sostenible o Desarrollo Sustentable, formulado en 1987 por la Comisión Mundial de Medio Ambiente y Desarrollo (CMMAD), por medio de su informe Nuestro Futuro Común, cuando plantea:

El desarrollo sostenible es el desarrollo que satisface las necesidades de la generación presente sin comprometer la capacidad de las generaciones futuras para satisfacer sus propias necesidades. Encierra en sí dos conceptos fundamentales:-el concepto

de "necesidades", en particular las necesidades esenciales de los pobres, a las que se debería otorgar prioridad; - la idea de limitaciones impuestas por el estado de la tecnología y la organización social entre la capacidad del Medio Ambiente 
para satisfacer las necesidades presentes y futuras [...] como exigencia mínima, el desarrollo duradero no debe poner en peligro los sistemas naturales que sostienen la vida en la Tierra: la atmósfera, las aguas, los suelos y los seres viviente (CMMAD, 1992: 29 y 76).

\section{LOS LATINOAMERICANOS RECOGEN Y ADOPTAN EL CONCEPTO DE ECODESARROLLO}

Lo interesante es que este concepto de ecodesarrollo, así entendido, lo hicieron inmediatamente suyo figuras destacadas del debate medioambiental latinoamericano de aquellos ańos, igualmente empeñados en entender la problemática ambiental desde la perspectiva del medio ambiente y desarrollo.

Según Enrique Leff, quien fuera discípulo de Sachs en la École des Hautes Études en Sciences Sociales, "las primeras propuestas sobre el ecodesarrollo encontraron en América Latina un territorio propicio para su promoción", de hecho, "el propio Ignacy Sachs consideraba a América Latina la región potencialmente más fértil para acoger sus propuestas y durante los años 70 viajó a varios países -principalmente a México y a Brasil, país en el que tenía vínculos de segunda ciudadanía- para promover el ecodesarrollo" (Leff, 2009: 221, 222). Por su parte, Gudynas señala que "la idea del ecodesarrollo, formalizada a mediados de los ańos setenta por Ignacy Sachs", tuvo una influencia limitada, "pero alcanzó visibilidad especialmente en América Latina” (Gudynas, 2011: 115).

La promoción del ecodesarrollo en AML, tuvo su apogeo entre 1972 y 1982, vale decir, "entre la Conferencia de Estocolmo sobre el Medio Ambiente Humano y la crisis financiera de América Latina" (Leff, 1998: 167). En estos años, figuras como Iván Restrepo, Enrique Leff, Vicente Sánchez, Héctor Sejenovich, Jaime Hurtubia, Francisco Szekely, Francisco Mieres, Hilda Herzer, Margarita Merino de Botero, Gilberto Gallopin, Pablo Gutman, Jorge Morello, e incluso Enrique Iglesias, entre muchos otros, recogieron este discurso dado que vinculaba muy bien la temática de unir en un solo concepto las ideas de medio ambiente y desarrollo. Estos intelectuales y científicos latinoamericanos, estaban vinculados principalmente a la CEPAL y al PNUMA, así como a institutos dedicados al estudio de los recursos naturales y a la problemática medioambiental de sus países, tales como el Instituto para el Desarrollo de los Recursos Naturales (INDERENA) de Colombia, o el grupo de trabajo de Sociedad y Naturaleza de la CLACSO, o la propia fundación del Centro de Ecodesarrollo de México. Todos ellos van a comenzar a trabajar muy tempranamente, la problemática ambiental sobre la base de este concepto de ecodesarrollo (INDERENA, 1976; Sejenovich, 2013). 
Por ejemplo, volvamos al ya mencionado seminario realizado en agosto de 1973 en México, "Medio Ambiente y Desarrollo: Estrategias para el Tercer Mundo", en el cual Sachs expuso su concepto de ecodesarrollo por primera vez y apenas un año después de finalizada la Conferencia de Estocolmo de 1972. En este seminario, junto a destacados intelectuales mexicanos, participaron varios altos funcionarios del gobierno del presidente Luis Echeverría, tanto secretarios de Estado como funcionarios responsables de la planificación del desarrollo. El propósito del seminario, era "promover al nivel más alto de toma de decisiones la incorporación de la dimensión ambiental en las prácticas del desarrollo en México", al punto que este evento se convirtió "prácticamente en 'una visita de Estado', en la que Sachs se reunió con varios ministros y con el mismo presidente Echeverría (Leff, 1998: 167). Y, entre los resultados más importantes de este encuentro fue la creación del Centro de Ecodesarrollo (ECODES), desde donde "se desarrollaron las primeras investigaciones orientadas a diagnosticar los problemas socio-ambientales del país y a generar propuestas para un desarrollo acorde con las condiciones ecosistémicas y socioambientales de México" (Leff, 2009: 222).

Así, la influencia de Sachs en AML fue inmediata. En enero de 1975, Leff publicó, "Hacia un proyecto de ecodesarrollo", para plantear la necesidad de aplicar en países latinoamericanos y del Tercer Mundo estilos de desarrollo que estuvieran de acuerdo con sus realidades físicas que condicionan la naturaleza y los recursos, así como sus realidades socio-culturales e históricas, ya que de esta forma se podían tener mejores rendimientos energéticos y productivos asegurando un desarrollo a largo plazo, y esto era mucho mejor que la trasplantación mecánica de los estilos de desarrollo primermundistas unilineales que se transformaban, en la AML y el Tercer Mundo, en malas imitaciones de lo que ocurría en los países desarrollados (Leff, 1975: 84-92). Posteriormente, en 1977, volverá a plantear, respecto del ecodesarrollo:

No se trata ni de un conservacionismo cultural y ecológico, ni de sustituir el conocimiento tradicional por la ciencia y tecnología moderna, producto de la racionalidad capitalista. El ecodesarrollo no es un proyecto social fundado en el eficientísimo energético o calórico de una nueva filosofía tecnocrática. Por el contrario, el objetivo del ecodesarrollo puede definirse como una serie de acciones conducentes a crear los conocimientos y las técnicas necesarias para el aprovechamiento de los recursos de cada ecosistema dentro de los criterios ecológicos que garantizan su reproducción. La selección de los recursos y los usos a los que se asigna su producción siguen estando determinados por los distintos proyectos sociales en que se define esta estrategia de ecodesarrollo (Leff, 1977a: 107).

Igualmente, coordinó el "Primer Simposio Sobre Ecodesarrollo", realizado en la UNAM en noviembre de 1976, en el cual "confluyeron científicos de muy 
diversas disciplinas para reflexionar y debatir la forma como la crisis ambiental emergente podría relacionarse con sus campos de conocimiento", y en donde "se expresó la idea de que la crisis ambiental era recurrente de las formas de conocimiento, de la racionalidad económica, del logocentrismo de la ciencia" (Leff, 2009: 224). Las ponencias de este Simposio dieron origen a una publicación homónima (Leff, 1977b).

De igual forma, se debe destacar el trabajo del argentino Héctor Sejenovich, que teniendo el concepto de ecodesarrollo como idea matriz, impulsó la creación de un grupo de estudio sobre el tema de medio ambiente y desarrollo en América Latina al interior de CLACSO. Esta idea se discutió y aprobó en la reunión de la Comisión Rural de la CLACSO realizada en 1977 en Costa Rica (reunión que estuvo dirigida por el sociólogo chileno Hugo Zemelman). De esta forma surgió el grupo Sociedad y Naturaleza al interior de la Comisión de Desarrollo Urbano y Regional de CLACSO, que comenzó a funcionar en 1978 a cargo del propio Sejenovich. Este grupo, Sociedad y Naturaleza, llegó a tener representantes de diez países latinoamericanos, entre ellos Víctor Toledo y Fernando Ortiz de México, Mauricio Rubirosa e Hilda Herzer de Argentina, Francisco Mieres de Venezuela y Daniel Pomarico de Uruguay entre muchos otros (Sejenovich, 2013).

De la misma manera, el chileno Vicente Sánchez, en su calidad de director de la Oficina Regional para América Latina y el Caribe del PNUMA (ORPALC, 1975-78), impulsó la socialización del este concepto en la región. Entre otras acciones, en 1978, o sea nueve años antes de la publicación del informe Nuestro Futuro Común que desarrolló en extenso el concepto de desarrollo sustentable, Vicente Sánchez junto con Héctor Sejenovich, Jaime Hurtubia y Francisco Skzekely, señalaban que "el objetivo básico y central del ecodesarrollo es utilizar los recursos para la satisfacción de las necesidades de la población, asegurando un mejoramiento de la calidad de vida de las generaciones actuales y futuras" (Sánchez, et al, 1978: 24). E, igualmente, en otro artículo escrito esta vez solo con Sejenovich, también en 1978, volvía a señalar:

Consideramos al ecodesarrollo como una modalidad del desarrollo económico que postula la utilización de los recursos para la satisfacción de las necesidades de las actuales y futuras generaciones de la población, mediante la maximización de la eficiencia funcional de los ecosistemas a largo plazo, empleando una tecnología adecuada a este fin y la plena utilización de las potencialidades humanas, dentro de un esquema institucional que permita la participación de la población en las decisiones fundamentales (Sánchez y Sejenovich, 1978: 154).

También, Sánchez y Sejenovich fueron compiladores del libro Antología en torno al ecodesarollo, publicado en 1983 en donde se planteaban temas que iban 
desde la importancia del turismo y el medio ambiente en AML, la conservación del patrimonio cultural como parte del tema de protección ambiental, hasta la conservación de los recursos naturales y la implementación de la energía solar, entre otros (Sánchez y Sejenovich, 1983).

Igualmente, este concepto de ecodesarrollo orientó el amplio estudio de diagnóstico y de proyecto de políticas públicas venezolano, Sistemas Ambientales para la Planificación, implementado con el apoyo de la ORPALC y del que habría de surgir el primer Ministerio del Medio Ambiente creado en AML, en 1978 (Leff, 2009; Sánchez, 2011).

En 1983, con apoyo de la ORPALC, se publicó el libro colombiano Ecodesarrollo, el pensamiento del decenio, en donde diferentes autores van a enfocar el tema del medio ambiente y desarrollo en AML desde la perspectiva del ecodesarrollo. Por ejemplo, Enrique Iglesias escribió el capítulo "Pasado, presente y futuro del ecodesarrollo", señalado que, "contrariamente a la tendencia a mirar el problema ecológico como algo negativo, como un costo que hay que absorber, está cada vez más claro que hay formas de desarrollo económico que se ven beneficiadas netamente por un manejo adecuado del medio ambiente" y, por este motivo, desde la CEPAL se concentraban en transmitir a "los gobiernos de la región que el manejo del medio ambiente interesa no sólo con fines de preservación ecológica, sino que también es un factor económico positivo que se puede sumar a otros" (Iglesias, 1983: 510).

Cabe mencionar también el apoyó que prestó, tanto a profesionales como a distintos gobiernos latinoamericanos, el Centro Internacional de Formación en Ciencias Ambientales de España (CIFCA), para avanzar en el tratamiento y solución de los problemas ambientales de la región desde la perspectiva del ecodesarrollo. Creado en 1975 por el gobierno español con apoyo del PNUMA, hasta su cierre en 1983, fue la institución que según Leff, "quizá con más fuerza estimuló el desarrollo de un pensamiento iberoamericano y latinoamericano a través de los seminarios que organizó y de sus publicaciones" (Leff, 2009: 224). Por ejemplo, entre sus apoyos más destacados a los estudios sobre ecodesarrollo en AML cabe mencionar: Una Experiencia de Ecodesarrollo. El caso de Santa Marta, Colombia (CIFCA, 1978); La Formación Ambiental en América Latina (CIFCA, 1978); La Tecnología latinoamericana. Seminario sobre nutrición y vivienda (CIFCA, 1979); Medio Ambiente y Urbanización (1982); así como la obra se síntesis, Diez años después de Estocolmo (CIFCA, 1983).

Sin embargo, este concepto de ecodesarrollo fue declinando en su uso en AML en la misma medida que se popularizaba el concepto de desarrollo sustentable definido por la CMMAD, que si bien publicó su informe Nuestro Futuro Común en 1987, desde 1984 comenzó trabajar en la idea de conciliar definitivamente los conceptos de medio ambiente y desarrollo. Como plantea Leff, el discurso de la 
sustentabilidad desplazó al de ecodesarrollo y lo dejó aprisionado de "los juegos del poder por la apropiación de la naturaleza y la gobernabilidad del mundo" (Leff, 1998: 169).

\section{A MODO DE CONCLUSIÓN}

Sin duda que en los tempranos ańos del debate ambiental en la agenda política mundial, el concepto de ecodesarrollo, estructurado por Ignacy Sachs, fue un enorme aporte e influencia para los intentos de los teóricos de AML, que bregaban por hacer confluir los conceptos de medio ambiente y desarrollo. E, igualmente, ayudó a acercar la problemática ambiental a quienes participaban de la planificación del desarrollo en esta región del mundo.

Fue un concepto que apoyó los esfuerzos desplegados por la CEPAL, el PNUMA y su oficina regional ORPALC, a la CLACSO, así como a institutos nacionales dedicados al estudio de los recursos naturales, como el colombiano INDERENA. Estas instituciones se esforzaron en los años 70 e inicios de los 80, por sumarse a la discusión ambiental global, desde una a perspectiva propia, que recogiera las realidades ecológicas, culturales y sociales de la región y reorientara los modelos de desarrollo incorporando la variable ambiental en toda su complejidad.

De aquí entonces, se puede plantear la hipótesis de que el ecodesarrollo fue una temprana idea en el naciente debate ambiental que, haciéndose cargo de la crisis ambiental, vino a contestar las tesis primermundistas que, absolutizando los límites físicos de la tierra, culpaban por la generación de la crisis ambiental, al crecimiento demográfico -sobre todo en el Tercer Mundo-, así como consideraban que la industrialización y el crecimiento económico - del cual ellos eran sus principales beneficiarios-, debía detenerse. Tesis que, en un mundo tan desigual en cuanto a riqueza, calidad de vida y oportunidades de desarrollo humano, causaron rechazo en el Tercer Mundo en general y en AML en particular.

Por lo tanto, el ecodesarrollo fue una forma de expresar una idea que era central para el Tercer Mundo, como era unir desarrollo y medio ambiente. En este sentido, fue recogido y trabajado por los profesionales y teóricos de AML, muchos de ellos expertos en temas del desarrollo, que participaban en el debate ambiental que había surgido en el seno de la sociedad internacional.

Por otra parte, sin duda que tras la acuńación del concepto de desarrollo sustentable por la CMMAD y su amplia socialización a partir de 1987, el ecodesarrollo salió del horizonte discursivo de quienes participaban de este debate. Cierto es que el desarrollo sustentable y el ecodesarrollo no son conceptos idénticos, pero mucho de los planteamientos formulados por el ecodesarrollo fueron recogidos en el concepto definido por la comisión Bruntland, de hecho algunos de los 
latinoamericanos difusores del concepto del ecodesarrollo formaron parte de esta Comisión, como fue el caso del chileno Vicente Sánchez (Sánchez, 2011) y, en este sentido, el ecodesarrollo al unir tempranamente las ideas de medio ambiente y desarrollo, fue precursor del concepto de desarrollo sustentable.

\section{REFERENCIAS}

Bifani, Paolo. Medio Ambiente y Desarrollo Sostenible. Madrid: Instituto de Estudios Políticos Para América Latina, 1999.

CIFCA. Una Experiencia de Ecodesarrollo. El caso de Santa Marta, Colombia. Madrid: CIFCA, 1978.

CIFCA. La Formación ambiental en América Latina. Madrid: CIFCA, 1978.

CIFCA. La Tecnología latinoamericana. Seminario sobre nutrición y vivienda. Madrid: CIFCA, 1979.

CIFCA, ClACSO. Medio Ambiente y Urbanización. Buenos Aires: CLACSO, 1982.

CIFCA. Diez años después de Estocolmo. Madrid: CIFCA, 1983.

Comisión Mundial del Medio Ambiente y del Desarrollo (CMMD); Nuestro Futuro Común. Madrid: Alianza Editorial, 1992.

Ehrlich, Paul R. The Population Bomb. 1ª Edition. New York: Ballantine Books, 1968.

Engfeldt, Lars-Göran. From Stockholm to Johannesburg and beyond. Stockholm: Government Offices of Sweden, Ministry of Foreign Affairs, 2009.

Estenssoro, Fernando. Medio Ambiente e Ideología. La Discusión pública en Chile, 1992-2002. Antecedentes para una historia de las ideas politicas a inicios del siglo XXI. Santiago de Chile: USACH / Ariadna, 2009.

"El Informe de Founex". En Margarita Marino de Botero y Juan Tokatlian (comps.), Ecodesarrollo. El pensamiento del decenio. Bogotá: IDERENA/ PNUMA, (1983): 51-85. 
Grinberg, MigueL. Ecofalacias. De cómo las multinacionales se apoderan del discurso del ambiente. Buenos Aires: Galerna, 1999.

Gudynas, Eduardo."Ambiente, sustentabilidad y desarrollo: una revisión de los encuentros y desencuentros”. En Javier Reyes Ruiz y Elba Castro Rosales (coords.), Contornos educativos de la sustentabilidad. México: Editorial Universitaria, Universidad de Guadalajara, (2011): 109-144.

Guimaraes, Roberto. "El discreto encanto de la cumbre de la tierra. Evaluación impresionista de Río 92", Nueva Sociedad 122 (1992): 86-103.

Gutiérrez Garza, Esthela y González Gaudiano, Édgard. De las Teorías del Desarrollo al Desarrollo Sustentable. México D.F.: Siglo XXI-UANL, 2010.

Herzer, Hilda et al., "La relación entre el hombre y los recursos naturales. Algunas consideraciones teóricas acerca del medio ambiente en AL", Nueva Sociedad 31-32, (1977): 206-220.

Iglesias, Enrique V. "Pasado, presente y futuro del ecodesarrollo". En Margarita Marino de Botero y Juan Tokatlian (comps.), Ecodesarrollo, el pensamiento del decenio. Bogotá: INDERENA/PNUMA, (1983): 507-512.

Instituto para el Desarrollo de los Recursos Naturales de Colombia (INDERENA); Términos de referencia para el estudio de factibilidad del proyecto piloto de ecodesarrollo en el macizo de Santa Marta. Bogotá: INDERENA, 1976.

Leff, Enrique. "Hacia un proyecto de ecodesarrollo", Comercio Exterior, XXV, 1, (1975): 84-92.

Leff, Enrique. "Etnobotánica, biosociología y ecodesarrollo", Nueva Antropología. volumen II /6, (1977a): 99-109.

Leff, Enrique. Primer Simposio Sobre Ecodesarrollo. México D.F.: Asociación Mexicana de Epistemología, UNAM, 1977 b.

Leff, Enrique. "Ignacy Sachs y el Ecodesarrollo". En Paulo Freire Vieira, Andrés Ribeiro Mauricio, Roberto Messias Franco y Renato Caporali Cordeiro (orgs.), Desenvolvimento e Meio Ambiente no Brasil. A contribuicão de Ignacy Sachs. Florianópolis: Editora Palloti/APED, (1998): 161-172. 
Leff, Enrique. "Pensamiento Ambiental Latinoamericano: Patrimonio de un Saber para la sustentabilidad". En VI Congreso Iberoamericano de Educación Ambiental. Buenos Aires: Secretaria de Ambiente y Desarrollo Sustentable, (2009): 215-236.

Mahrane, Yannick; Fenzi, Marianna; Pessis, Céline; Bonneuil, Christophe. "De la Nature à la Biosphère. L'invetion de l'enivironnement global, 1945-1972", Vingtième Siècle. Revue d'histoire, 113/1 (2012): 127-141.

Meadows, Dennis et al., The Limits to Growth. New York: A Potomac Associates Book, 1972.

Restrepo, Iván. "El Ecodesarrollo y algunos problemas agropecuarios", Comercio Exterior, 26/1 (1976): 9-16.

Sachs, Ignacy. "Ambiente y estilo de desarrollo", Comercio Exterior, XXIV, (1974): 360-368.

Sachs, Ignacy. "Los Limites: ¿Realidad o Fantasía? En Joseph Hodara, Iván Restrepo ¿Tiene límites el Crecimiento? Una visión latinoamericana. México D.F.: El manual Moderno, (1977): 12-22.

Sachs, Ignacy. "Ecodesarrollo: concepto, aplicación, beneficios y riesgos", Agricultura y Sociedad, 18, (1981): 9-32.

Sachs, Ignacy. "Do Crescimento Econômico ao Ecodesenvolvimento". En Paulo Freire Vieira, Andrés Ribeiro Mauricio, Roberto Messias Franco, Renato Caporali Cordeiro, Renato (organs.), Desenvolvimento e Meio Ambiente no Brasil. A contribuicão de Ignacy Sachs. Florianópolis: Editora Palloti/APED, (1998): 161-163.

Sánchez, Vicente. Recuerdos sobre el origen del debate ambiental. Santiago, julio de 2011. Entrevista otorgada a Fernando Estenssoro.

Sánchez, Vicente y Sejenovich, Héctor."Ecodesarrollo: Una estrategia para el desarrollo social y económico compatible con la conservación ambiental”, Revista Interamericana de Planificación, Volumen XII, Números 47-48, septiembre - diciembre, (1978): 152-160. 
Sánchez, Vicente; Sejenovich, Héctor; Szekely, Francisco y Hurtubia, Jaime. "Hacia Una conceptualización del ecodesarrollo". En CIFCA, Cuadernos del CIFCA $N^{\circ}$ 9: Una Experiencia de ecodesarrollo. El caso de Santa Marta, Colombia. Madrid: CIFCA, (1978): 12 -33.

Sánchez, Vicente y Sejenovich, Héctor. Antología en torno al ecodesarrollo. San José de Costa Rica: Universidad Estatal a Distancia, 1983.

Sejenovich, Héctor. Entrevista concedida a Fernando Estenssoro, en Santiago de Chile, junio de 2013 (Inédita).

Strong, Maurice. "El décimo aniversario de la Conferencia de Estocolmo". En Héctor Echechuri et al., Diez Años Después de Estocolmo. Desarrollo, Medio Ambiente y Supervivencia. Madrid: CIFCA, (1983): 243-253.

Ward, Barbará y Dubos, René. Una Sola Tierra. El cuidado y conservación de un pequeño planeta. México D. F.: Fondo de Cultura Económica, 1972. 